ELECTRONIC RESEARCH ANNOUNCEMENTS OF THE AMERICAN MATHEMATICAL SOCIETY

Volume 3, Pages 45-51 (May 20, 1997)

S $1079-6762(97) 00022-\mathrm{X}$

\title{
NONLINEAR PARABOLIC PROBLEMS ON MANIFOLDS, AND A NONEXISTENCE RESULT FOR THE NONCOMPACT YAMABE PROBLEM
}

\author{
QI S. ZHANG
}

(Communicated by Richard Schoen)

\begin{abstract}
We study the Cauchy problem for the semilinear parabolic equations $\Delta u-R u+u^{p}-u_{t}=0$ on $\mathbf{M}^{n} \times(0, \infty)$ with initial value $u_{0} \geq 0$, where $\mathbf{M}^{n}$ is a Riemannian manifold including the ones with nonnegative Ricci curvature. In the Euclidean case and when $R=0$, it is well known that $1+\frac{2}{n}$ is the critical exponent, i.e., if $p>1+\frac{2}{n}$ and $u_{0}$ is smaller than a small Gaussian, then the Cauchy problem has global positive solutions, and if $p<1+\frac{2}{n}$, then all positive solutions blow up in finite time. In this paper, we show that on certain Riemannian manifolds, the above equation with certain conditions on $R$ also has a critical exponent. More importantly, we reveal an explicit relation between the size of the critical exponent and geometric properties such as the growth rate of geodesic balls. To achieve the results we introduce a new estimate for related heat kernels.

As an application, we show that the well-known noncompact Yamabe problem (of prescribing constant positive scalar curvature) on a manifold with nonnegative Ricci curvature cannot be solved if the existing scalar curvature decays "too fast" and the volume of geodesic balls does not increase "fast enough". We also find some complete manifolds with positive scalar curvature, which are conformal to complete manifolds with positive constant and with zero scalar curvatures. This is a new phenomenon which does not happen in the compact case.
\end{abstract}

\section{INTRODUCTION}

We shall announce new results in the study of the global existence and blow up of the following semilinear parabolic Cauchy problem:

$$
\left\{\begin{array}{l}
H u \equiv H_{0} u+u^{p}=\Delta u-R u-\partial_{t} u+u^{p}=0 \quad \text { in } \quad \mathbf{M}^{n} \times(0, \infty), \\
u(x, 0)=u_{0}(x) \geq 0 \quad \text { in } \quad \mathbf{M}^{n}
\end{array}\right.
$$

where $\mathbf{M}^{n}$ with $n \geq 3$ is a noncompact complete Riemannian manifold, $\Delta$ is the Laplace-Beltrami operator, and $R=R(x)$ is a bounded function.

Throughout the paper we make the following assumptions.

(i) There are positive constants $b, C$, and $K$ such that

$$
|B(x, r)| \leq C r^{n} ; \quad|B(x, 2 r)| \leq C 2^{n}|B(x, r)|, r>0 ; \quad \text { Ricci } \geq-K .
$$

Received by the editors February 19, 1997.

1991 Mathematics Subject Classification. Primary 35K55; Secondary 58G03.

(C) 1997 American Mathematical Society 
(ii) $G$, the fundamental solution of the linear operator $H_{0}=\Delta-R-\partial_{t}$ in (1.1), has global Gaussian upper bound, i.e.,

$$
0 \leq G(x, t ; y, s) \leq \frac{C}{\left|B\left(x,(t-s)^{1 / 2}\right)\right|} e^{-b \frac{d(x, y)^{2}}{t-s}},
$$

for all $x, y \in \mathbf{M}^{n}$ and all $t>s$.

(iii) When $d(x, y)^{2} \leq t-s, G$ satisfies

$$
G(x, t ; y, s) \geq \min \left\{\frac{1}{C\left|B\left(x,(t-s)^{1 / 2}\right)\right|}, \frac{1}{C\left|B\left(y,(t-s)^{1 / 2}\right)\right|}\right\} .
$$

If one assumes that the Ricci curvature of $\mathbf{M}^{n}$ is nonnegative and $R=0$, it is well known that that above properties hold (see $[\mathrm{LY}]$ ). More general conditions on $\mathbf{M}^{n}$ (without the nonnegativity of Ricci) that support (1.2), (1.3), and (1.3') can be found in $[\mathrm{G}]$. When $R$ is not identically zero, $(1.3)$ and $\left(1.3^{\prime}\right)$ pose certain restrictions on $R$ as indicated in Corollary 2.3 below.

Equation (1.1) with $R=0$ contains the following important special cases. When the Riemannian metric is just the Euclidean metric, (1.1) becomes the semilinear parabolic equation which has been studied by many authors. In the paper $[\mathrm{Fu}]$, Fujita proved the following results:

(a) when $1<p<1+\frac{2}{n}$ and $u_{0}>0$, problem (1.1) possesses no global positive solutions;

(b) when $p>1+\frac{2}{n}$ and $u_{0}$ is smaller than a small Gaussian, then (1.1) has global positive solutions. So $1+\frac{2}{n}$ is the critical exponent.

For a rich literature on the subsequent development of the topic, we refer the reader to the survey paper [Le].

In recent years many authors have undertaken the research on semilinear elliptic operators on manifolds, including the well-known Yamabe problem (see [Sc] and [Yau]). In contrast, not much has been done for their semilinear parabolic counterpart. It looks more imperative to fill this gap when one takes into account the tremendous literature about the heat kernel of a complete Riemannian manifold, which is still a linear theory. The study of Ricci flows also leads to semilinear parabolic problems (see $[\mathrm{H}]$ and $[\mathrm{Sh}]$ ). We shall need some new techniques to study when blow up of solutions occur, and when global positive solutions exist for equation (1.1). In fact the prevailing methods in treating semilinear problems are variational and comparison method. The variational approach seems hard to apply when the manifold is noncompact. The comparison method also faces some difficulties on curved manifolds because the intrinsic operators are not of constant coefficients.

The method we are using is based on some new inequalities involving the heat kernels. We are able to reveal an explicit relation between the size of the critical exponent and geometric properties of the manifold such as the growth rate of geodesic balls (see Theorem B).

It is interesting to note that Theorem B immediately leads to a nonexistence result for the well-known noncompact Yamabe problem of prescribing positive constant scalar curvatures, i.e., whether the following elliptic problem has positive solutions on $\mathbf{M}^{n}$ (see Theorem C):

$$
\Delta u-\frac{n-2}{4(n-1)} R u+u^{(n+2) /(n-2)}=0 .
$$

This problem has been posed by J. Kazdan $[\mathrm{K}]$ and S. T. Yau [Yau]. The compact version of the problem was proposed by Yamabe [Yam], proved by Trudinger [Tr] 
and Aubin $[\mathrm{Au}]$ in some cases, and eventually proved by R. Schoen [Sc] completely. In the noncompact case, Aviles and McOwen $[\mathrm{AM}]$ obtained some existence result for the problem of prescribing constant negative scalar curvature. However, as far as we know, there has been no result on the problem of prescribing constant positive scalar curvature, which was termed more difficult in $[\mathrm{AM}]$.

When $\mathbf{M}^{n}$ is $\mathbf{R}^{n}$ with the Euclidean metric, then problem (1.4) becomes $\Delta u+$ $u^{(n+2) /(n-2)}=0$, which does have positive solutions (see [Ni])

$$
u_{\lambda}(x)=\frac{\left[n(n-2) \lambda^{2}\right]^{(n-2) / 4}}{\left(\lambda^{2}+|x|^{2}\right)^{(n-2) / 2}}, \quad \lambda>0 .
$$

So it is reasonable to expect that (1.4) has a solution. However, Theorem C below asserts that unlike the compact Yamabe problem, problem (1.4) cannot be solved in general. In fact, if the existing scalar curvature decays "too fast" and the volume of geodesic balls does not increase "fast enough", then (1.4) has no solution.

Before stating the main results explicitly we list some notation.

Definition 1.1. A function $u=u(x, t)$ such that $u \in L_{l o c}^{2}\left(\mathbf{M}^{n} \times(0, \infty)\right)$ is called a solution of (1.1) if

$$
u(x, t)=\int_{\mathbf{M}^{n}} G(x, t ; y, 0) u_{0}(y) d y+\int_{0}^{t} \int_{\mathbf{M}^{n}} G(x, t ; y, s) u^{p}(y, s) d y d s
$$

for all $(x, t) \in \mathbf{M}^{n} \times(0, \infty)$.

$G=G(x, t ; y, s)$ will denote the fundamental solution of the linear operator $H_{0}$ in (1.1). For any $c>0$, we write

$$
G_{c}(x, t ; y, s)=\left\{\begin{array}{l}
\frac{1}{\left|B\left(x,(t-s)^{1 / 2}\right)\right|} \exp \left(-c \frac{d(x, y)^{2}}{t-s}\right), \quad t>s, \\
0, \quad t<s .
\end{array}\right.
$$

Let $u_{0}$ be a positive function in $L^{\infty}\left(\mathbf{M}^{n}\right)$ and $a>0$; we write

$$
\begin{aligned}
h_{a}(x, t) & =\int_{\mathbf{M}^{n}} G_{a}(x, t ; y, 0) u_{0}(y) d y ; \\
h(x, t) & =\int_{\mathbf{M}^{n}} G(x, t ; y, 0) u_{0}(y) d y .
\end{aligned}
$$

Given $V=V(x, t)$ and $c>0$, we introduce the notation

$$
\begin{aligned}
N_{c, \infty}(V) \equiv & \sup _{x \in \mathbf{M}^{n}, t>0} \int_{-\infty}^{\infty} \int_{\mathbf{M}^{n}}|V(y, s)| G_{c}(x, t ; y, s) d y d s \\
& +\sup _{y \in \mathbf{M}^{n}, s>0} \int_{\infty}^{\infty} \int_{\mathbf{M}^{n}}|V(x, t)| G_{c}(x, t ; y, s) d x d t .
\end{aligned}
$$

We note that $N_{c, \infty}(V)$ may be infinite for some $V$. However, the fact that $N_{c, \infty}(V)$ is finite for some specific functions will play a key role in the proof of the theorems.

\section{MAin Results}

The main results of the paper are the next three theorems. 
Theorem A. Suppose $R=0$ and (1.2), (1.3), and $\left(1.3^{\prime}\right)$ hold; the critical exponent of (1.1) is $p^{*}=1+\frac{1}{s^{*}}$, where

$$
\begin{aligned}
& s^{*}=\sup \left\{s \mid \lim _{t \rightarrow \infty} \sup t^{s}\|W(\cdot, t)\|_{L^{\infty}}<\infty,\right. \\
& \left.\quad \text { for a nonnegative and nontrivial } W \text { such that } H_{0} W=0\right\} .
\end{aligned}
$$

Remark 2.1. Theorem A, which establishes the existence of the critical exponent, can be proved by following the proof of Theorem 1 in [Me]. However, this theorem does not provide any estimate of the size of the exponent. It does not even tell whether $p^{*}$ is closer to 1 or $\infty$. Our main concern is therefore to find an explicit relation between the critical exponent and geometrical properties of the manifold. This is done in the next

Theorem B. Let $\mathbf{M}^{n}$ be any Riemannian manifold and $R=R(x)$ any bounded functions such that (1.2), (1.3), and $\left(1.3^{\prime}\right)$ hold; then the following conclusions are true.

(a) Suppose, for $t>s \geq 0$,

$$
\sup _{x \in \mathbf{M}^{n}, t} \int_{r_{0}}^{\infty} \int_{\mathbf{M}^{n}} \frac{G_{c}(x, t ; y, s)}{\left|B\left(y, s^{1 / 2}\right)\right|^{p-1}} d y d s+\sup _{y \in \mathbf{M}^{n}, s} \int_{r_{0}}^{\infty} \int_{\mathbf{M}^{n}} \frac{G_{c}(x, t ; y, s)}{\left|B\left(x, t^{1 / 2}\right)\right|^{p-1}} d x d t<\infty
$$

for some $r_{0}>0$ and a suitable $c>0$; then (1.1) has global positive solutions for some $u_{0} \geq 0$.

(b) Suppose for some $x_{0} \in \mathbf{M}^{n}$,

$$
\lim _{r \rightarrow \infty} \inf \frac{\left|B\left(x_{0}, r\right)\right|}{r^{\alpha}}<\infty
$$

then (1.1) has no global positive solutions for any $p<1+\frac{2}{\alpha}$ and any $u_{0} \geq 0$.

To exemplify the conditions in Theorem B, we provide two corollaries.

Corollary 2.1. Under the same assumptions as in Theorem B, suppose

$$
\int_{r_{0}}^{\infty} \sup _{x \in \mathbf{M}^{n}} \frac{1}{\left|B\left(x, r^{1 / 2}\right)\right|^{p-1}} d r<\infty
$$

for some $r_{0}>0$; then (1.1) has global positive solutions for some $u_{0} \geq 0$. In particular, if, for an $\alpha>0$, $\inf _{x \in \mathbf{M}^{n}}|B(x, r)| \geq C r^{\alpha}$ when $r$ is sufficiently large, then for $p>1+\frac{2}{\alpha}$, (1.1) has global positive solutions for some $u_{0}$.

In the next corollary, we show that if $\mathbf{M}^{n}$ has bounded geometry in the sense of E. B. Davies (see p. 172 in [D]), which means the existence of a function $b(r)$ and $c>0$ such that

$$
c^{-1} b(r) \leq|B(x, r)| \leq c b(r)
$$

for all $x \in \mathbf{M}^{n}$ and $r>0$, then the critical exponent of (1.1) can be explicitly determined.

Corollary 2.2. Suppose, in addition to the assumptions in Theorem B, (2.1) holds; then $p^{*}$, the critical exponent of $(1.1)$, is given by $p^{*}=1+\frac{2}{\alpha^{*}}$, where

$$
\alpha^{*}=\inf \left\{\alpha>0 \mid \lim _{r \rightarrow \infty} \inf \frac{|B(x, r)|}{r^{\alpha}}<\infty, x \in \mathbf{M}^{n}\right\} \text {. }
$$

Remark 2.2. By (2.1), the above number $\alpha^{*}$ is independent of the choice of $x \in \mathbf{M}^{n}$. 
Remark 2.3. Under the assumptions of part (a) of Theorem B, (1.1) has global positive solutions if $u_{0} \geq 0$ satisfies

$$
u_{0} \in C^{2}\left(\mathbf{M}^{n}\right), \quad \lim _{d(x, 0) \rightarrow \infty} u_{0}(x)=0, \quad \text { and } \quad\left\|u_{0}\right\|_{L^{\infty}\left(\mathbf{M}^{n}\right)}+\left\|u_{0}\right\|_{L^{1}\left(\mathbf{M}^{n}\right)} \leq b_{0},
$$

where $b_{0}$ is a sufficiently small number and 0 is a point in $\mathbf{M}^{n}$. This result is new even in the Euclidean case.

Next we turn our attention to the noncompact Yamabe problem of prescribing positive scalar curvatures.

Theorem C. Suppose (1.2), (1.3), and (1.3') hold and

$$
\lim _{r \rightarrow \infty} \inf \frac{\left|B\left(x_{0}, r\right)\right|}{r^{\alpha}}<\infty
$$

for some $x_{0}$ and $\alpha<(n-2) / 2$; then the Yamabe problem (1.4) has no solutions.

It is important to know what conditions should be imposed on the Ricci curvature and the scalar curvature $R$ so that the conditions of Theorem $\mathrm{C}$ be met. To this end we have

Corollary 2.3. (a) Suppose the Ricci curvature of $\mathbf{M}^{n}$ is nonnegative, and for a suitable $c>0, N_{c, \infty}(R)$ is sufficiently small; then (1.2), (1.3), and (1.3') hold. Hence the Yamabe problem has no solutions if (2.2) holds.

(b) In particular, suppose $\mathbf{M}^{n}$ is a manifold with nonnegative Ricci curvature and $|B(x, r)| \sim r^{\alpha}$ for $2<\alpha<(n-2) / 2$ and large $r>0$. Then if

$$
0 \leq R(x) \leq \epsilon /\left(1+d^{2+\delta}(x, 0)\right)
$$

for a sufficiently small $\epsilon$ and $a \delta>0$, then the Yamabe problem (1.4) has no solution. Here 0 is a point in $\mathbf{M}^{n}$. An example is $\mathbf{M}^{9}=\mathbf{R}^{3} \times S^{1} \times \cdots \times S^{1}$ with the metric tensor being the direct product of those usual ones on $\mathbf{R}^{3}$ and $S^{1}$.

Remark 2.4. In the above example, we can just change the metric in $\mathbf{R}^{3}$ suitably so that the scalar curvature of $\mathbf{M}^{9}$ be positive but (1.4) still have no solution. Indeed, by part (b) of the corollary, any metric $g^{\prime}$ on $\mathbf{R}^{3}$ satisfying the next three conditions can be a choice: (i) $\left|B\left(x^{\prime}, r\right)\right| \sim r^{3}, x^{\prime} \in \mathbf{R}^{3}$; (ii) Ricci is nonnegative; (iii) the scalar curvature $R^{\prime} \leq \epsilon /\left(1+d^{2+\delta}\left(x^{\prime}, 0\right)\right)$ for $x^{\prime} \in \mathbf{R}^{3}, \delta>0$, and a small $\epsilon>0$.

Remark 2.5. We would like to point out another fundamental difference between the noncompact Yamabe problem and the compact one. In the compact case, no manifold with positive scalar curvature is conformal to a manifold with zero scalar curvature. This is reflected from the fact that the equation $\Delta u-R u=0$ with $R>0$ has no positive solution on compact manifolds. However, this is not the case for noncompact manifolds. The following is a complete noncompact manifold which is conformal to a complete manifold of constant positive scalar curvature, and to a complete manifold with zero scalar curvature. Let $\mathbf{M}=S^{3} \times \mathbf{R}^{1}$ with the metric being the direct product of the usual ones on $S^{3}$ and $\mathbf{R}^{1}$. Then $R=6$, $n=4$ and hence equation (1.4) becomes

$$
\Delta u-u+u^{3}=0,
$$

which has a solution $u=1$. At the same time the equation

$$
\Delta u-u=0
$$


has a positive solution

$$
u\left(x_{1}, x_{2}\right)=e^{x_{2}}+e^{-x_{2}} \geq 1,
$$

where $x_{1} \in S^{3}$ and $x_{2} \in \mathbf{R}^{1}$. Clearly the above solution generates a complete metric of zero scalar curvature since it is bounded away from zero. Similar phenomenon can be shown for $\mathbf{M} \times \mathbf{R}^{k}$ where $\mathbf{M}$ is any compact manifold with positive scalar curvature (see [Zhang5]).

Let us briefly discuss the method we are going to adopt. We will use the Schauder fixed point theorem to achieve existence. This requires some new estimates involving the heat kernel on $\mathbf{M}^{n}$, such as, for $0<a<b$ and $s<\tau<t$,

(i)

$$
G_{a}(x, t ; z, \tau) G_{b}(z, \tau ; y, s) \leq C\left[G_{c}(x, t ; z, \tau)+G_{c}(z, \tau ; y, s)\right] G_{a}(x, t ; y, s),
$$

$$
G_{b}(x, t ; z, \tau) G_{a}(z, \tau ; y, s) \leq C\left[G_{c}(x, t ; z, \tau)+G_{c}(z, \tau ; y, s)\right] G_{a}(x, t ; y, s) .
$$

(iii) Suppose $0<a<b$; there exist positive constants $C_{a, b}$ and $c$ depending only on $a$ and $b$ such that, for all $t>s \geq 0$,

$$
\begin{gathered}
\int_{s}^{t} \int_{\mathbf{M}^{n}} G_{a}(x, t ; z, \tau)|V(z, \tau)| G_{b}(z, \tau ; y, s) d z d \tau \leq C_{a, b} N_{c, \infty}(V) G_{a}(x, t ; y, s) ; \\
\int_{s}^{t} \int_{\mathbf{M}^{n}} G_{b}(x, t ; z, \tau)|V(z, \tau)| G_{a}(z, \tau ; y, s) d z d \tau \leq C_{a, b} N_{c, \infty}(V) G_{a}(x, t ; y, s) .
\end{gathered}
$$

To prove Theorem C, the key idea is to obtain global bounds (1.3) and $\left(1.3^{\prime}\right)$ for the fundamental solution of $H_{0}$, which involves certain conditions on the scalar curvature. Then we show that the parabolic problem (1.1) with $p=\frac{n+2}{n-2}$ has no global positive solutions under the assumptions of Theorem C. Since every positive solution of the Yamabe equation (1.4) is a global positive solution of the parabolic problem (1.1), the former cannot exist either.

Details of the proof can be found in the paper [Zhang5].

\section{ACKNOWLEDGMENT}

I should thank Professor J. Beem for helpful discussions, and Professors N. Garofalo, H. A. Levine, and Z. Zhao for their interest and support.

\section{REFERENCES}

[A] D.G. Aronson, Nonnegative solutions of linear parabolic equations, Annali della Scuola Norm. Sup. Pisa 22 (1968), 607-694. MR 55:8553

[Au] T. Aubin, The scalar curvature, Differential geometry and relativity, edited by Cahen and Flato, Reidel, Dordrecht, 1976, pp. 5-18. MR 55:6476

[AM] P. Aviles and R. C. McOwen, Conformal deformation to constant negative scalar curvature on noncompact Riemannian manifolds, J. Diff. Geometry 27 (1988), 225-239. MR 89b:58225

[D] E. B. Davies, Heat kernels and spectral theory, Cambridge Univ. Press, Cambridge and New York, 1989. MR 90e:35123

[Fu] H. Fujita, On the blowing up of solutions of the Cauchy problem for $u_{t}=\Delta u+u^{1+\alpha}$, J. Fac. Sci. Univ. Tokyo, Sect. I 13 (1966), 109-124. MR 35:5761 
[G] A. A. Grigoryan, The heat equation on noncompact Riemannian manifolds, Mat. Sb. 182 (1991), 55-87; English transl., Math. USSR Sb. 72 (1992), 47-77. MR 92h:58189

[H] R. Hamilton, Three-manifolds with positive Ricci curvature, J. Diff. Geom. 17 (1982), 255-306. MR 84a:53050

[K] J. Kazdan, Prescribing the curvature of a Riemannian manifold, Amer. Math. Soc., Providence, RI, 1985. MR 86h:53001

[Le] H. A. Levine, The role of critical exponents in blowup theorems, SIAM Review 32 (1990), 269-288. MR 91j:35135

[LN] Tzong-Yow Lee and Wei-Ming Ni, Global existence, large time behavior and life span of solutions of a semilinear parabolic Cauchy problem, Transactions AMS 333 (1992), 365-378. MR 92k:35134

[LSU] O. A. Ladyzhenskaya, V. A. Solonnikov, and N. N. Uraltseva, Linear and quasilinear equations of parabolic type, Transl. Math. Monographs, vol. 23, Amer. Math. Soc., Providence, RI, 1968. MR 39:3159b

[LY] P. Li and S. T. Yau, On the parabolic kernel of the Schrödinger operator, Acta Math. 156 (1986), 153-201. MR 87f:58156

[Me] P. Meier, On the critical exponent for reaction-diffusion equations, Arch. Rat. Mech. Anal. 109 (1990), 63-71. MR 90k:35038

[Mo] J. Moser, A Harnack inequality for parabolic differential equations, Comm. Pure and Appl. Math. 17 (1964), 101-134. MR 28:2357

[Ni] W. M. Ni, On the elliptic equation $\Delta u+K(x) u^{(n+2) /(n-2)}=0$, its generalizations and applications in geometry, Indiana Univ. Math. J. 31 (1982), 493-529. MR 84e:35049

[Sa] L. Saloff-Coste, A note on Poincaré, Sobolev and Harnack inequality, IMRN, Duke Math. J. 2 (1992), 27-38. MR 93d:58158

[Sc] R. Schoen, Conformal deformation of a Riemannian metric to constant scalar curvature, J. Diff. Geometry 20 (1984), 479-495. MR 86i:58137

[Sh] W.-X. Shi, Deforming the metric on complete Riemannian manifolds, J. Diff. Geometry 30 (1989), 225-301. MR 90i:58202

[Tr] N. Trudinger, Remarks concerning the conformal deformation of Riemannian structures on compact manifolds, Ann. Scuola Norm. Sup. Pisa Cl. Sci. (4) 22 (1968), 265-274. MR 39:2093

[Yam] H. Yamabe, On a deformation of Riemannian structures on compact manifolds, Osaka Math. J. 12 (1960), 21-37. MR 23:A2847

[Yau] S. T. Yau, Seminar on differential geometry, Annals of Math. Studies, vol. 102, Princeton Univ. Press, Princeton, NJ 1982. MR 83a:53002

[Zhao] Z. Zhao, On the existence of positive solutions of nonlinear elliptic equations - A probabilistic potential theory approach, Duke Math. J. 69 (1993), 247-258. MR 94c:35090

[Zhang1] Qi Zhang, On a parabolic equation with a singular lower order term, Transactions of AMS 348 (1996), 2811-2844. MR 96k:35073

[Zhang2] Qi Zhang, On a parabolic equation with a singular lower order term, Part II, Indiana U. Math. J., to appear.

[Zhang3] Qi Zhang, Global existence and local continuity of solutions for semilinear parabolic equations, Comm. PDE, to appear.

[Zhang4] Qi Zhang, The critical exponent of a reaction-diffusion equation on some Lie groups, Math. Z., to appear.

[Zhang5] Qi Zhang, Semilinear parabolic problems on manifolds and applications to the noncompact Yamabe problem, preprint.

Department of Mathematics, University of Missouri, Columbia, MO 65211

E-mail address: sz@mumathnx6.math.missouri.edu 